\title{
Vulnerability of Parkinson Patients to Iatrogenic Adverse Events: Emphasis on the Perioperative Period through a Case Report
}

\author{
Catherine Maurice ${ }^{1,2 *}$, Kiran Grant ${ }^{3}$, Austin M. Pereira ${ }^{3}$ and Yasser B. Abulhasan ${ }^{2,3,4}$ \\ ${ }^{1}$ Division of Neurology, Department of Medicine, Pencer Brain Tumor Centre, Princess Margaret Cancer Centre, University Health Network - Univer- \\ sity of Toronto, Toronto, Ontario, Canada \\ ${ }^{2}$ Neurocritical Care Unit, Division of Neurology, Department of Medicine, Montréal Neurological Institute, McGill University, Montréal, Québec, \\ Canada \\ ${ }^{3}$ Faculty of Medicine, University of Toronto, Toronto, Ontario, Canada \\ ${ }^{4}$ Faculty of Medicine, Health Sciences Center, Kuwait University, Kuwait
}

${ }^{*}$ Corresponding author: Catherine Maurice, MD, FRCPC - Neuro-Oncologist, Princess Margaret Cancer, Centre - Pencer Brain Cancer Centre, 610 University Avenue, Office 18-717, Toronto, ON, Canada; Office Phone: 416-946-2277; Fax: 416-946-2284; Email: Catherine.Maurice@uhn.ca

Received: December 22, 2019; Accepted: January 22, 2019; Published: January 24 2019;

\begin{abstract}
Deep brain stimulator surgery represents an interesting option for medically resistant Parkinson's disease patients, indisposed by "wearing-off" and motor fluctuations. Considering the neurodegenerative nature of PD, after approximately five years of natural disease evolution, one half to twothirds of patients develops this delayed complication. The occurrence of dyskinesia and motor fluctuations interferes with activities of the daily living, consequently impacting the quality of life. A high frequency chronic stimulation targeting the subthalamic nucleus, located ventral to the thalamus and involved in the basal ganglia system, generates the same outcome than an ablation of this structure. Despite the positive impact of DBS surgery on PD symptomatology, physicians should be mindful of the vulnerability of this particular population and promote thorough monitoring, especially during the perioperative phase. A thoughtful approach considering the pathophysiological mechanisms underlying PD is required in order to select an individual's pharmacopoeia. We report the case of a 61 year-old male, known for seventeen year of evolving PD, who developed acutely a state of generalized symmetrical rigidity, with a predilection for axial and bulbar musculature. After a meticulous review of this patient's pharmacologic profile, sufentanil was deemed the responsible agent.
\end{abstract}

Keywords: Sufentanil, Parkinson Disease; Deep Brain Stimulator, Wearing-Off, Motor Fluctuations, Dopamine

\section{Introduction}

After several years of Parkinson's Disease (PD) evolution, most patients develop a wearing-off effect or motor fluctuations induced by chronic exposure to exogenous levodopa. Despite the introduction of dopaminergic agonists, Catechol-O-Methyl Transferase Inhibitors (COMTI) or amantadine, the efficacy of the Deep Brain Stimulator (DBS) on this delayed complication is undeniable. On the other hand, PD patients are prone to serious adverse events justifying cautious choices in the selection of an anesthetic regimen, especially in the perioperative period.

\section{Case Description}

A 61 year-old male, known for seventeen year of evolving PD, underwent bilateral deep brain stimulator implantation. The surgery comports three systematic steps. The first stage consisted of a right temporal lead implantation. This procedure is repeated on the contralateral side on the following day. Finally, the last stage consists in the stimulator internalization.
The preoperative neurologic examination revealed a severe resting tremor implicating bilateral upper and lower limbs. The speech was significantly compromised by the facial rigidity and bradykinesia. Supplemental pertinent findings included: chin tremor, diffuse and global appendicular cogwheel rigidity, postural instability and a typical parkinsonian gait. While our patient was "en route" to the operation room (OR), he was administered his regular dopaminergic regimen. An uneventful general anesthesia involved the following agents: sufentanil, propofol, rocuronium, and lidocaine. No intra-operative antidopaminergic medication was judged necessary. Upon arrival to ICU (Intensive Care Unit), our gentleman developed an acute severe extrapyramidal reaction immediately following extubation. The main concern at this point was the limitation of thoracic expansion, at imminent risk to evolve towards respiratory failure. The administration of benzodiazepines was insufficient to reach stable respiratory parameters, justifying an emergent reintubation. The acute iatrogenic reaction induced dysphagia, preventing the administration of oral medication such as levodopacarbidopa, mandating the installation of a nasogastric tube. A direct correlation was observed between the improved chest wall compliance and the expected washout period of opioids. 


\section{Discussion}

PD, described initially as the "Shaky Palsy" by James Parkinson in the $19^{\text {th }}$ century, affects $0.3 \%$ of the overall population [1]. The endpoint of PD consists of progressive vanishing of the dopaminergic neurons within the substantia nigra pars compacta. This process induces an imbalance between dopaminergic inhibition and cholinergic excitation of the striatal output. Dopaminergic depletion subsequently induces excessive thalamic inhibition. The phenotypic repercussion of dopamine depletion is classical "parkinsonism", characterized by: akinesia/bradykinesia, rigidity, tremor and postural instability.

Electrophysiological mapping mandatorily requires the patient's cooperation. Medication preventing hyperkinetic symptoms is contraindicated in the immediate postsurgical state. The priority is to depict those involuntary movements, to assure a proper positioning of the leads and confirm the success of the procedure. Leads implantation is performed in two distinct steps. Firstly, the stereotaxic frame and leads are positioned. Local analgesia such as a scalp bloc is preferred to general anaesthesia, to maintain the patient's cooperation. General anesthesia is performed during the second stage, consisting of the internalization of the leads and implantation of the programmable impulse generator.

Every anaesthetic agent comports pros and cons, an individualized "case-by-case" approach is preferable. Opioids should be administered cautiously in general, and even more in parkinsonian individuals considering PD's pathophysiological basis. The striatum, representing a major source of input to the basal ganglia, contains a high concentration of opioid receptors, modulating reward, addiction and motor control [2]. Opioids neuropeptides, such as enkephalin and dynorphin. contribute to neuromodulation. A divergent form of plasticity leads to the remodeling of the nigrostriatal network, as a consequence of dopamine depletion and its treatment: exogenous $L$-dopa. The outcome is an upgrade of opioid transmission involving the basal ganglia, possibly preventing the development of motor dyskinesias [3]. In parallel, fentanyl and sufentanil are powerful opioids reported to induce a rare complication: "The Wooden Chest Syndrome" [4]. Several hypotheses were emitted to explain the opioid-induced rigidity resulting from narcotic exposure. PD patients are particularly vulnerable, considering their unique intrinsic features, mentioning alteration in the central mu1-opioid receptors, cerulospinal noradrenergic and glutamatergic pathways, as well as spinal motoneurons [5] (Table 1). This complication can le lethal, as increased muscular tone incapacitates the closure of the vocal cords, glottis and jaw, potentially accompanied by thoraco-abdominal rigidity impairing ventilation.

\section{Conclusion}

In conclusion, DBS surgery could be a life changing procedure for medically resistant PD candidates selected thoroughly based on their symptomatology, and realistic expectations. Anaesthetic drugs should be prescribed judiciously to prevent deleterious iatrogenic events. The importance of this case relies on three main factors: 1) the increasing popularity of DBS surgery, 2) the high prevalence of PD in our society, and 3) the high likelihood for any individual to necessitate general anesthesia in the context of a surgical procedure in a lifetime. Those elements highlight the necessity to develop optimal knowledge regarding perioperative pharmacotherapy in $\mathrm{PD}$ context.

Table 1. Hypothetical Pathways and Mechanisms Predisposing Parkinson's Disease Patients to Develop "Wooden Chest Syndrome.

\begin{tabular}{|l|l|}
\hline \multicolumn{1}{|c|}{ Structure/Pathway } & \multicolumn{1}{c|}{ Mechanism } \\
\hline Striatal opioid binding sites & Interaction with dopaminergic D2-receptors. \\
\hline Neurotransmitters imbalance & $\begin{array}{l}\text { Reduced dopamine level results in imbalance } \\
\text { with the cholinergic neurotransmitter system; } \\
\text { both systems impact the control of muscle tone, } \\
\text { leads to rigidity. }\end{array}$ \\
\hline Striatal dopaminergic neurons & $\begin{array}{l}\text { Dopamine depletion results in nigro-striatal } \\
\text { dysfunction }\end{array}$ \\
\hline $\begin{array}{l}\text { Inhibition of tyrosine } \\
\text { hydroxylase in the nigro- } \\
\text { striatal system }\end{array}$ & $\begin{array}{l}\text { Induced opioids, tyrosine hydroxylase is an } \\
\text { essential enzyme acting on dopamine sysnthesis. } \\
\text { Overall impact is increased muscle tone/rigidity. }\end{array}$ \\
\hline $\begin{array}{l}\text { Decline in pallidal GABA } \\
\text { level }\end{array}$ & $\begin{array}{l}\text { Due to interconnection of the dopaminergic- } \\
\text { gabaminergic systems, decline in the pallidal } \\
\text { GABA concentration. Overall impact is } \\
\text { activation of cholinergic neurons projecting to } \\
\text { thalamus. }\end{array}$ \\
\hline $\begin{array}{l}\text { Docus ceruleus descending } \\
\text { signal } \\
\text { concentration in the putamen }\end{array}$ & $\begin{array}{l}\text { Impact of opioids: reduced gabaminergic } \\
\text { transmission, improved by benzodiazepines. }\end{array}$ \\
\hline Pons descending signal & $\begin{array}{l}\text { Cerulospinal noradrenergic and glutamatergic } \\
\text { impact of spinal neurons. }\end{array}$ \\
\hline $\begin{array}{l}\text { Increase in efferent motor signal, leading to } \\
\text { Activation of spinal motoneurones. }\end{array}$ \\
\hline
\end{tabular}

\section{Highlights}

1. The endpoint of Parkinson's disease is a progressive vanishing of the dopaminergic neurons within the "substantia nigra pars compacta". The clinical picture resulting from this ongoing depletion is named "parkinsonism", characterized by: akinesia/ bradykinesia, rigidity, tremor and postural instability.

2. Despite the introduction of dopaminergic agonists, catecholO-methyl transferase inhibitors or amantadine, the efficacy of subthalamic deep brain stimulator implantation is undeniable to improve the course of dyskinesia and motor fluctuations, typically presenting after several years of disease evolution.

3. High frequency chronic stimulation targeting the subthalamic nucleus generates the same outcome than an ablation of this structure, explaining the effectiveness of deep brain stimulator implantation in medically resistant Parkinson's disease patients.

4. Deep brain stimulator surgery represents an interesting option for medically resistant Parkinson Disease patients, indisposed by "wearing-off" and motor fluctuations, considering the neurodegenerative nature of Parkinson's disease, resulting in incurable, indolent and progressive symptoms.

5. It is essential to have in mind the pathophysiological mechanisms underlying Parkinson's disease in order to select optimally given patient's pharmacopoeia. 
6. Parkinson's disease patients are particularly vulnerable during the perioperative phase. Several risk factors are linked to the modifications of the pharmacologic profile during that period. Local or generalized anaesthesia represent a supplemental risk of developing an iatrogenic adverse events or drug interaction.

\section{Author Contribution}

Catherine Maurice: Conceptualization, Drafting, Redaction, Supervision, Editing, Revision and Final Approval.

Kiran Grant: Redaction, Editing, Revision and Final Approval.

Austin M. Pereira: Redaction, Editing, Revision and Final Approval.

Yasser B. Abulhasan: Conceptualization, Drafting, Redaction, Supervision, Editing, Revision and Final Approval.

\section{Abbreviations}

DBS: Deep Brain Stimulator

PD: Parkinson's Disease

COMT: Catechol-O-Methyltransferase

CNS: Central Nervous System

\section{References}

1. J. Parkinson (1817) An essay on the Shaking Palsy. J Neuropsychiatry and Clinical Neurosciences 14: 223-236

2. A.H. Erga, I. Dalen, A. Ushakova, J. Chung, C. Tzoulis, et al (2018) Dopaminergic and Opioid Pathways Associated with Impulse Control Disorders in Parkinson's Disease. Front Neurol 9:109

3. MA Cenci (2007) Dopamine Dysregulation of Movement Control in 1-DOPAInduced Dyskinesia. Trends Neurosci 30: 236-243. [Crossref]

4. C.P. Phua, A. Wee, A. Lim, J. Abisheganaden, A. Verma (2017) Fentanyl-Induced Chest Wall Rigidity Syndrome in a Routine Bronchoscopy. Respir Med Case Rep 20: 205-207.

5. V. Dimitriou, I. Zogogiannis, D. Liotiri, F. Wambi, N. Tawfeeq, et al (2014) Impossible Mask Ventilation After an Unusually Dose Fentanyl-Induced Muscle Rigidity in a Patient with Essential Tremor: A Case Report and Review of the Literature. Middle East J Anaesthesiol 22: 619-622. 